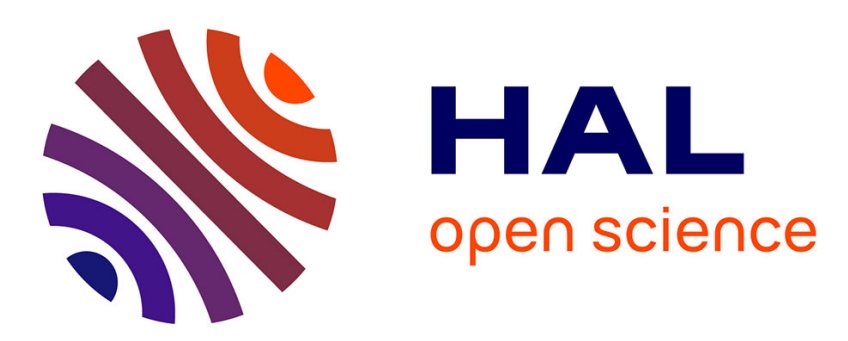

\title{
An Implicit Integral Formulation for the Modeling of Inviscid Fluid Flows in Domains Containing Obstacles
}

Clément Colas, Martin Ferrand, Jean-Marc Hérard, Erwan Le Coupanec, Xavier Martin

\section{- To cite this version:}

Clément Colas, Martin Ferrand, Jean-Marc Hérard, Erwan Le Coupanec, Xavier Martin. An Implicit Integral Formulation for the Modeling of Inviscid Fluid Flows in Domains Containing Obstacles. Finite Volumes for Complex Applications 8, Jun 2017, Lille, France. pp.53 - 61, 10.1007/978-3-319-573946_6. hal-01579372

\author{
HAL Id: hal-01579372 \\ https://hal.science/hal-01579372
}

Submitted on 31 Aug 2017

HAL is a multi-disciplinary open access archive for the deposit and dissemination of scientific research documents, whether they are published or not. The documents may come from teaching and research institutions in France or abroad, or from public or private research centers.
L'archive ouverte pluridisciplinaire HAL, est destinée au dépôt et à la diffusion de documents scientifiques de niveau recherche, publiés ou non, émanant des établissements d'enseignement et de recherche français ou étrangers, des laboratoires publics ou privés. 


\title{
An Implicit Integral Formulation for the Modeling of Inviscid Fluid Flows in Domains Containing Obstacles
}

\author{
Clément Colas, Martin Ferrand, Jean-Marc Hérard, Erwan Le Coupanec and \\ Xavier Martin
}

\begin{abstract}
We focus here on an integral approach to compute compressible inviscid fluid flows in physical domains cluttered up with many small obstacles. This approach is based on a multidimensional porous integral formulation of Euler system of equations. Its discretization uses a first order semi-implicit finite volume scheme with pressure-correction algorithm preserving the positivity of both density and pressure. Numerical tests are completed by simulating a 2D channel flow containing two aligned tubes. The results are compared to reference solutions obtained with a pure fluid approach on a fine mesh.
\end{abstract}

Key words: Finite volumes, integral formulation, porous media, compressible flow

\section{Introduction}

In this paper we introduce a way to investigate fluid flows in thermohydraulic circuits components in nuclear reactors where three so-called "system", "component" and "local" representation scales coexist. The first one is a 0D/1D description and aims at providing a real time simulation of full circuits. The third one is the CFD scale and allows a fine description on restricted physical domains. The intermediate scale relies on a homogenized representation of some components [7, 9]; it consists in taking into account a fluid and solid volume in cells. Our purpose is to build a

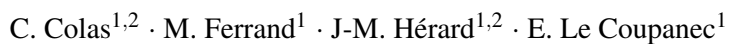

${ }^{1}$ EDF R\&D, MFEE, 6 quai Watier, 78400, Chatou, France

${ }^{2}$ Aix-Marseille Université, I2M, UMR CNRS 7373, 39 rue Joliot Curie, 13453, Marseille, France e-mail: clement.colas@edf.fr, martin.ferrand@edf.fr, jean-marc.herard@edf.fr, erwan.lecoupanec@edf.fr

X. Martin

IFP Énergies nouvelles, 1 et 4 avenue du Bois Prau, 92852, Rueil-Malmaison, France

e-mail: xavier.martin@ifpen.fr 
formulation embedding the "local" and "component" scales and ensuring the continuity between these two scales. A possible approach has been introduced in [6] using explicit schemes. The basic idea consists in an integral formulation of PDEs in a domain where a fluid flows around many small obstacles. Herein an implicit finite volume scheme is considered, using the open-source code Code_Saturne [4]. The compressible Euler equations (1) governing inviscid fluid flows are considered, and the unknowns $\rho, \boldsymbol{u}, P$ respectively denote the density, the velocity and the pressure of the fluid, while the momentum is $\boldsymbol{Q}=\rho \boldsymbol{u}$. The volumetric total energy $E$ is such that $E=\rho\left(\frac{u^{2}}{2}+\varepsilon(P, \rho)\right)$. The internal energy $\varepsilon(P, \rho)$ is prescribed by the EOS (Equation Of State), $\boldsymbol{f}$ is a mass volumetric external force and $\Phi_{v}$ a volumetric heat transfer source term. Thus the set of governing equations is:

$$
\left\{\begin{array}{l}
\partial_{t} \rho+\nabla \cdot \boldsymbol{Q}=0 \\
\partial_{t} \boldsymbol{Q}+\nabla \cdot(\boldsymbol{u} \otimes \boldsymbol{Q})+\nabla P=\rho \boldsymbol{f} \\
\partial_{t} E+\nabla \cdot(\boldsymbol{u}(E+P))=\rho \boldsymbol{f} \cdot \boldsymbol{u}+\rho \Phi_{v}
\end{array}\right.
$$

The speed of acoustic waves noted $c$ is such that: $c^{2}=\left(\frac{P}{\rho^{2}}-\frac{\partial \varepsilon(P, \rho)}{\partial \rho}\right) /\left(\frac{\partial \varepsilon(P, \rho)}{\partial P}\right)$. The total enthalpy is: $H=\frac{E+P}{\rho}$, and $\boldsymbol{W}$ is the conservative variable: $\boldsymbol{W}=(\rho, \boldsymbol{Q}, E)^{t}$.

\section{Integral Formulation}

The integral form of conservation laws described in $[8,6]$ is considered. Set of equations (1) is integrated on control volumes $\Omega_{i}$ which may contain many solid obstacles. All $\Omega_{i}$ cells form a mesh of the computational domain $\Omega \subset \mathbb{R}^{d}(d=1,2$ or 3), such that: $\bar{\Omega}=\cup_{i} \bar{\Omega}_{i}$. The obstacles may be completely or partially included in $\Omega_{i}$. Part of a control volume boundary may coincide with the surface of an obstacle. Fig.1 is a sketch of the admissible situtations. In the sequel, the subscript $i j$ refers to interfaces between neighbouring control volumes $\Omega_{i}$ and $\Omega_{j}$, and the superscript $\phi$ refers to fluid volumes and interfaces $i j$ where the fluid may cross the interface, noted $\Gamma_{i j}^{\phi}$ of measure $S_{i j}^{\phi}=$ meas $\left(\Gamma_{i j}^{\phi}\right)$. Besides, the superscript $w$ refers to solid interfaces where a wall boundary $\Gamma_{i}^{w}$ of measure $S_{i}^{w}$ is located inside the control volume $\Omega_{i}$ or on its boundary. The mass flux is null through surfaces $S_{i}^{w}$. The volume occupied by the fluid within the control volume $\Omega_{i}$ is denoted by $\Omega_{i}^{\phi}$. Nonetheless, a control volume $\Omega_{i}$ may contain several fluid sub-domains $\Omega_{i, k}^{\phi}(k \in \llbracket 1, N(i) \rrbracket$ with $N(i)$ the number of sub-elements), that are not connected to each other. We introduce within each fluid sub-cell $\Omega_{i, k}^{\phi}$ a mean value of the fluid state variable $\boldsymbol{W}(\mathbf{x}, t)$ noted $\boldsymbol{W}_{i, k}(t)$. The mean fluid state variable in cell $\Omega_{i}, \boldsymbol{W}_{i}(t)$, is introduced as follows:

$$
\operatorname{meas}\left(\Omega_{i}^{\phi}\right) \boldsymbol{W}_{i}(t)=\sum_{k \in\{1, \cdots, N(i)\}} \int_{\Omega_{i, k}^{\phi}} \boldsymbol{W}(\mathbf{x}, t) d x
$$


Fig. 1 A (blue) control volume $\Omega_{i}$ includes (gray) obstacles numbered from 1 to 5. Obstacles may: overlap part of the boundary of cell $i$ (1); partially occupy one fluid cell (or subcell) (2); and split it into two fluid sub-cells $\Omega_{i, 1}^{\phi}$ and $\Omega_{i, 2}^{\phi}$ (3); be totally included in cell $i$ or one of its subcells (4); be aligned with part of the boundary of cell $i$ (5). The dashed blue surface corresponds to the fluid-fluid part of the boundary $\Gamma_{i, k}^{\phi}$ between sub-cells $\Omega_{i, k}$ and their neighbouring sub-cells occupied by the fluid.

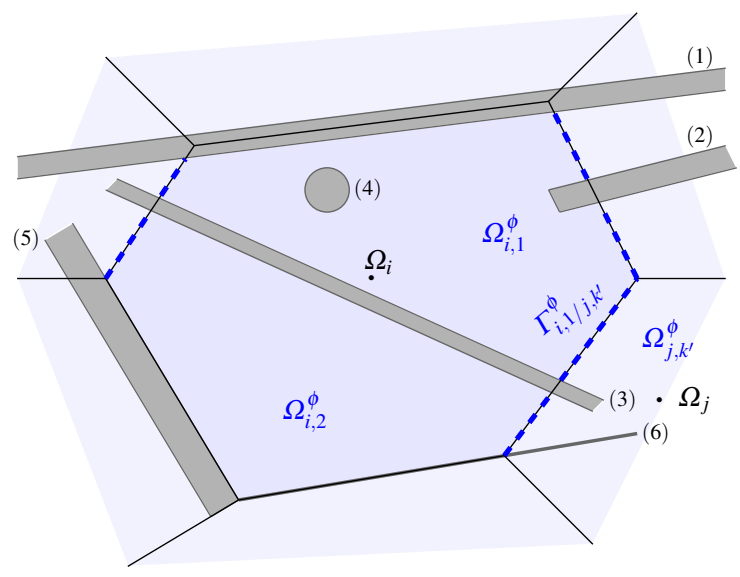

By additivity, using $\Omega_{i}^{\phi}=\bigcup_{k \in\{1, \cdots, N(i)\}} \Omega_{i, k}^{\phi}$, where $\Omega_{i, k}^{\phi}$ are all mutually disjoint:

$$
\operatorname{meas}\left(\Omega_{i}^{\phi}\right)=\sum_{k \in\{1, \cdots, N(i)\}} \operatorname{meas}\left(\Omega_{i, k}^{\phi}\right)
$$

The conservation laws (1) can be rewritten as follows:

$$
\partial_{t} \boldsymbol{W}+\boldsymbol{\nabla} \cdot \boldsymbol{F}(\boldsymbol{W})=\boldsymbol{D}(\boldsymbol{W})
$$

where $\boldsymbol{F}(\boldsymbol{W})=(\rho \boldsymbol{u}, \rho \boldsymbol{u} \otimes \boldsymbol{u}+P \mathbf{I}, \boldsymbol{u}(E+P))^{t}$ is the convective flux and $\boldsymbol{D}(\boldsymbol{W})=$ $\left(0, \rho \boldsymbol{f}, \rho\left(\boldsymbol{f} \cdot \boldsymbol{u}+\Phi_{v}\right)\right)^{t}$ represents the source term. Equation (2) is integrated over a bounded time interval $\left[t_{0}, t_{1}\right] \subset \mathbb{R}^{+}$and space with respect to the $\Omega_{i, k}^{\phi}$ sub-cell, the divergence theorem allows to rewrite:

$\int_{\Omega_{i, k}^{\phi}}\left(\boldsymbol{W}\left(\mathbf{x}, t_{1}\right)-\boldsymbol{W}\left(\mathbf{x}, t_{0}\right)\right) d x+\int_{t_{0}}^{t_{1}} \int_{\Gamma_{i, k}} \boldsymbol{F}(\boldsymbol{W}(\mathbf{x}, t)) \cdot \boldsymbol{n} d \Gamma d t=\int_{t_{0}}^{t_{1}} \int_{\Omega_{i, k}^{\phi}} \boldsymbol{D}(\boldsymbol{W}(\mathbf{x}, t)) d x d t$

Here, $\Gamma_{i, k}=\partial \Omega_{i, k}^{\phi}$ denotes the whole boundary of the fluid sub-cell $\Omega_{i, k}^{\phi}$ with $\boldsymbol{n}$ the outward normal vector. Fluid $\Gamma_{i, k}^{\phi}$ and wall $\Gamma_{i, k}^{w}$ boundaries of each sub-cell $\Omega_{i, k}^{\phi}$ are distinguished, such that: $\Gamma_{i, k}=\Gamma_{i, k}^{\phi} \cup \Gamma_{i, k}^{w}$ and $\Gamma_{i, k}^{\phi} \cap \Gamma_{i, k}^{w}=\emptyset$. Summing up over the $N(i)$ fluid sub-cells of the control volume $\Omega_{i}$, we get the integral formulation:

$$
\begin{aligned}
\operatorname{meas}\left(\Omega_{i}^{\phi}\right)\left(\boldsymbol{W}_{i}\left(t_{1}\right)-\boldsymbol{W}_{i}\left(t_{0}\right)\right) & +\sum_{k \in\{1, \cdots, N(i)\}} \int_{t_{0}}^{t_{1}} \int_{\Gamma_{i, k}^{\phi} \cup \Gamma_{i, k}^{w}} \boldsymbol{F}(\boldsymbol{W}(\mathbf{x}, t)) \cdot \boldsymbol{n} d \Gamma d t \\
& =\sum_{k \in\{1, \cdots, N(i)\}} \int_{t_{0}}^{t_{1}} \int_{\Omega_{i, k}^{\phi}} \boldsymbol{D}(\boldsymbol{W}(\mathbf{x}, t)) d x d t
\end{aligned}
$$




\section{Time Scheme}

The time discretization of the dynamic equation (4) is based on an implicit first order scheme. It is assumed that all numerical fluxes may be evaluated by means of a standard finite volume method, considering one mean value $\mathbf{W}_{\mathbf{i}}^{\mathbf{n}}$ per cell $\Omega_{i}$ at each time $t^{n} . \mathbf{W}_{\mathbf{i}}^{\mathbf{n}}$ is an approximation of $\boldsymbol{W}_{i}\left(t^{n}\right)$, and the time step at the $n^{t h}$ iteration is: $\Delta t^{n}=t^{n+1}-t^{n}$. The numerical algorithm uses a fractional step method, with prediction and correction of the pressure [5, 1]. Each time stepping is divided in three steps: a mass balance step which is used to update the density and to predict the pressure, a momentum balance step where the velocity is updated and an energy balance step that allows to update the total energy and to correct the pressure. The superscript $(\cdot)^{n+1,-}$ states that the variable is implicit for the current step (or known from the last step).

\section{Mass balance}

Pressure and density are implicit, while velocity and entropy are considered frozen at time $t^{n}$. The following scheme is set:

$$
\operatorname{meas}\left(\Omega_{i}^{\phi}\right) \frac{1}{\left(c^{2}\right)_{i}^{n}}\left(P_{i}^{n+1,-}-P_{i}^{n}\right)+\Delta t^{n} \int_{\Gamma_{i}^{\phi}} \boldsymbol{Q}^{*} \cdot \boldsymbol{n} d \Gamma=0
$$

where: $\left(c^{2}\right)^{n}=c^{2}\left(P^{n}, \rho^{n}\right)$, and the approximation $\delta P=\left(c^{2}\right)^{n} \delta \rho$ is considered, with $\delta P=P^{n+1,-}-P^{n}$. The approximation of the implicit mass flux $\boldsymbol{Q}^{*}$ is:

$$
Q^{*}=Q^{n}-\Delta t^{n} \nabla P^{n+1,-}
$$

and a two-point flux approximation is used: $\int_{\Gamma_{i}^{\phi}} \boldsymbol{\nabla} \phi \cdot \boldsymbol{n} d \Gamma=\sum_{j \in V(i)}\left(\phi_{j}-\phi_{i}\right) S_{i j}^{\phi} / h_{i j}$, on admissible meshes.

\section{Momentum balance}

In this step, velocity (and momentum) is implicit, whereas density and pressure are known from Equation (5), and total energy is frozen. Integration of the momentum equation gives:

$$
\begin{aligned}
\operatorname{meas}\left(\Omega_{i}^{\phi}\right)\left(\boldsymbol{Q}_{i}^{n+1,-}-\boldsymbol{Q}_{i}^{n}\right) & +\Delta t^{n} \int_{\Gamma_{i}^{\phi}}\left(\left(\boldsymbol{Q}^{*} \cdot \boldsymbol{n}\right) \boldsymbol{u}\right)^{n+1,-} d \Gamma+\Delta t^{n} \int_{\Gamma_{i}} P^{n+1,-} \boldsymbol{n d} \Gamma \\
& -\Delta t^{n} \operatorname{meas}\left(\Omega_{i}^{\phi}\right) \rho_{i}^{n+1,-} \boldsymbol{f}_{i}^{n+1,-}=0
\end{aligned}
$$

where: $P_{w}^{n+1,-}$ is equal to $P_{i}^{n+1,-}$ for all wall interfaces of cell $i$. This second step provides the velocity $\boldsymbol{u}^{n+1,-}$ and thus $\boldsymbol{Q}^{n+1,-}=\rho^{n+1,-} \boldsymbol{u}^{n+1,-}$, using:

$$
\left(\left(\boldsymbol{Q}^{*} \cdot \boldsymbol{n}\right) \boldsymbol{\phi}\right)_{i j}=\left(\boldsymbol{Q}^{*} \cdot \boldsymbol{n}\right)_{i j} \phi_{i j}^{u p w i n d}
$$

with: $\phi_{i j}^{u p w i n d}=\beta_{i j} \phi_{i}+\left(1-\beta_{i j}\right) \phi_{j}$, and: $\beta_{i j}=\max \left(0, \operatorname{sgn}\left(\boldsymbol{Q}^{*} \cdot \boldsymbol{n}\right)_{i j}\right)$. 


\section{Energy balance}

Total energy is implicit while pressure, density and velocity are explicit from the previous steps. Using upwind scheme (8), the total energy $E^{n+1,-}$ is updated:

$$
\begin{aligned}
\operatorname{meas}\left(\Omega_{i}^{\phi}\right)\left(E_{i}^{n+1,-}-E_{i}^{n}\right) & +\Delta t^{n} \int_{\Gamma_{i}^{\phi}}\left(\left(\boldsymbol{Q}^{*} \cdot \boldsymbol{n}\right) \frac{E+P}{\rho}\right)^{n+1,-} d \Gamma \\
& -\Delta t^{n} \operatorname{meas}\left(\Omega_{i}^{\phi}\right)\left(\rho \boldsymbol{f} \cdot \boldsymbol{u}+\rho \Phi_{v}\right)_{i}^{n+1,-}=0
\end{aligned}
$$

Property 1 (Positivity of the density and the pressure):

If the initial conditions are such that: $\rho_{i}^{n}>0$ and $P_{i}^{n}>0$ and the EOS is such that: $\hat{\gamma}=\rho c^{2} / P>1$. The density $\rho_{i}^{n+1,-}$ and the pressure $P_{i}^{n+1,-}$ remain positive for all $i$, if the time step $\Delta t^{n}$ complies with the CFL-like condition (10):

$$
\operatorname{meas}\left(\Omega_{i}^{\phi}\right) \geq \Delta t^{n} \sum_{j \in V(i)} \beta_{i j}\left(\frac{\rho_{i} c_{i}^{2}}{P_{i}}\right)^{n}\left(\boldsymbol{u}^{n} \cdot \boldsymbol{n}\right)_{i j} S_{i j}^{\phi}
$$

Sketch of proof. Equation (5) gives an invertible linear system: $\mathbf{A}\left(P_{i}^{n+1,-}\right)=\mathbf{B}$ with $\mathbf{A}^{-1} \geq 0$, and also $\mathbf{B} \geq 0$ if and only if condition (10) holds, thus implying $P_{i}^{n+1,-} \geq$ 0 . To be conservative, we have set: $\rho_{i}^{n+1,-}=\left(c^{-2}\right)_{i}^{n} P_{i}^{n+1,-}+\rho_{i}^{n}\left(\hat{\gamma}_{i}^{n}-1\right) / \hat{\gamma}_{i}^{n}$ which completes the proof: $\rho_{i}^{n+1,-} \geq 0$, since $\hat{\gamma}>1$.

Eventually, the variables are updated: $\rho^{n+1}=\rho^{n+1,-}, \boldsymbol{u}^{n+1}=\boldsymbol{u}^{n+1,-}, E^{n+1}=$ $E^{n+1,-}$, and $P^{n+1}=P\left(\rho^{n+1}, \varepsilon^{n+1}\right)$, where: $\rho^{n+1} \varepsilon^{n+1}=E^{n+1}-0.5 \rho^{n+1}\left(\boldsymbol{u}^{n+1}\right)^{2}$.

\section{Numerical Results}

\section{Mesh refinement impact}

Numerical approximations obtained thanks to this new approach are compared with approximate solutions of the fluid model when the mesh perfectly matches obstacles inside the computational domain (i.e. without any porous control volume). The integral approach is applied on porous meshes so that fluid cells are partially obstructed by obstacles. The numerical example consists in computing the steady flow of a compressible inviscid fluid in a channel aligned with the $x$-direction. At mid-length, the channel is cluttered by two identical, steady and impermeable tubes aligned with it. A sketch of the test case is displayed on Fig.2. The two-dimensional computational domain is $\Omega=[0, L] \times[0, h]$. It contains a discontinuous transition interface between a totally fluid area and an obstructed area at $x=\frac{L}{2}$. We consider admissible meshes, with faces aligned with the obstacles. At the inlet and outlet sections of the domain, boundary conditions from the resolution of half Riemann problems are enforced [3] and a steady state is computed. Slip wall boundary conditions are imposed at the top $(y=h)$ and bottom $(y=0)$ of the computational domain. The 
time step is controlled by the CFL-like condition (10). Several numerical approxi-

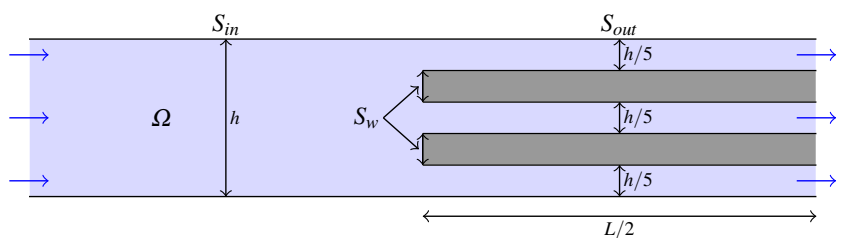

Fig. 2 The $\Omega$ domain has a $L \times h$ size, containing two internal obstacles (in gray). They lie in the downstream middle of $\Omega$ and are spaced of $\frac{h}{5}$ such that $S_{\text {out }}+S_{w}=S_{\text {in }}$. The fluid flows from the left inlet towards the right outlet.

mations of the steady state are obtained using coarse and fine meshes. Six meshes are perfectly adapted to the domain, thus including either totally fluid cells or fully solid cells. They are respectively composed of $24 \times 5,48 \times 10,96 \times 20,192 \times 40$, $384 \times 80$ and $768 \times 160$ regular cells. The four other meshes include porous cells, they are respectively composed of $24 \times 6,48 \times 12,96 \times 24$ and $192 \times 48$ regular cells. We assume that a steady state is reached when the dimensionless time residuals on pressure and velocity in $L^{2}$ norm become small enough $\left(\approx 10^{-7}\right.$, see Fig.3). The time to steadiness is mainly governed by the velocity time residual. We note $P^{w}$ the pressure on the intern upstream vertical faces, and $S^{w}$ the vertical wall surface of these intern upstream faces, such that $S_{w}=S_{\text {in }}-S_{\text {out }}$ (see Fig.2). We define the flux vector $\boldsymbol{\varphi}=[Q S, Q S H,(Q U+P) S]^{t}$ and the head losses vector $\boldsymbol{\Delta}=\left[0,0, P^{w} S_{w}\right]^{t}$, with $Q$ the momentum, $S$ the fluid cross section, $H$ the total enthalpy, $U$ the bulk velocity in the x-direction and $P$ the pressure. When the perfect steady state is reached, the conservation laws provide: $\boldsymbol{\varphi}_{\text {in }}=\boldsymbol{\varphi}_{\text {out }}+\boldsymbol{\Delta}$. The relative deviation between inlet and outlet boundary values for all the variables is defined as:

$$
e(\boldsymbol{\varphi})=\frac{\left|\boldsymbol{\varphi}_{\text {in }}-\left(\boldsymbol{\varphi}_{\text {out }}+\boldsymbol{\Delta}\right)\right|}{\left|\boldsymbol{\varphi}_{\text {in }}\right|+\left|\boldsymbol{\varphi}_{\text {out }}\right|+|\boldsymbol{\Delta}|} .
$$

For each mesh, $e(\boldsymbol{\varphi})$ is plotted for $\boldsymbol{\varphi}$ components on Fig.3. This deviation is small $\left(\leq 10^{-6}\right)$. When the cells number increases, $e(\boldsymbol{\varphi})$ may slightly increase since unsteady terms (vortices) appear on refined meshes.

\section{Mesh adaptation w.r.t. obstacles position: sensibility analysis}

The coarsest mesh, composed of $24 \times 5$ cells, is considered for two sensibility tests. The differences in results between the adapted fluid mesh and any configuration where the bottom of one of the obstacles is slightly shifted off its grid edge are compared. The first configuration, called $M_{1}$, corresponds to the mesh adapted to the tubes position. In the second configuration, $M_{2}$, the lower tube width is slightly reduced $\left(10^{-5} h\right)$ so that weakly porous cells exist. In the last situation, $M_{3}$, the width of the same tube is reduced again at the top $\left(10^{-5} h\right)$, and its upstream wall is also slightly shifted in the downstream direction $\left(10^{-5} h\right)$. The relative deviation, $E^{M_{k} M_{l}}$, 

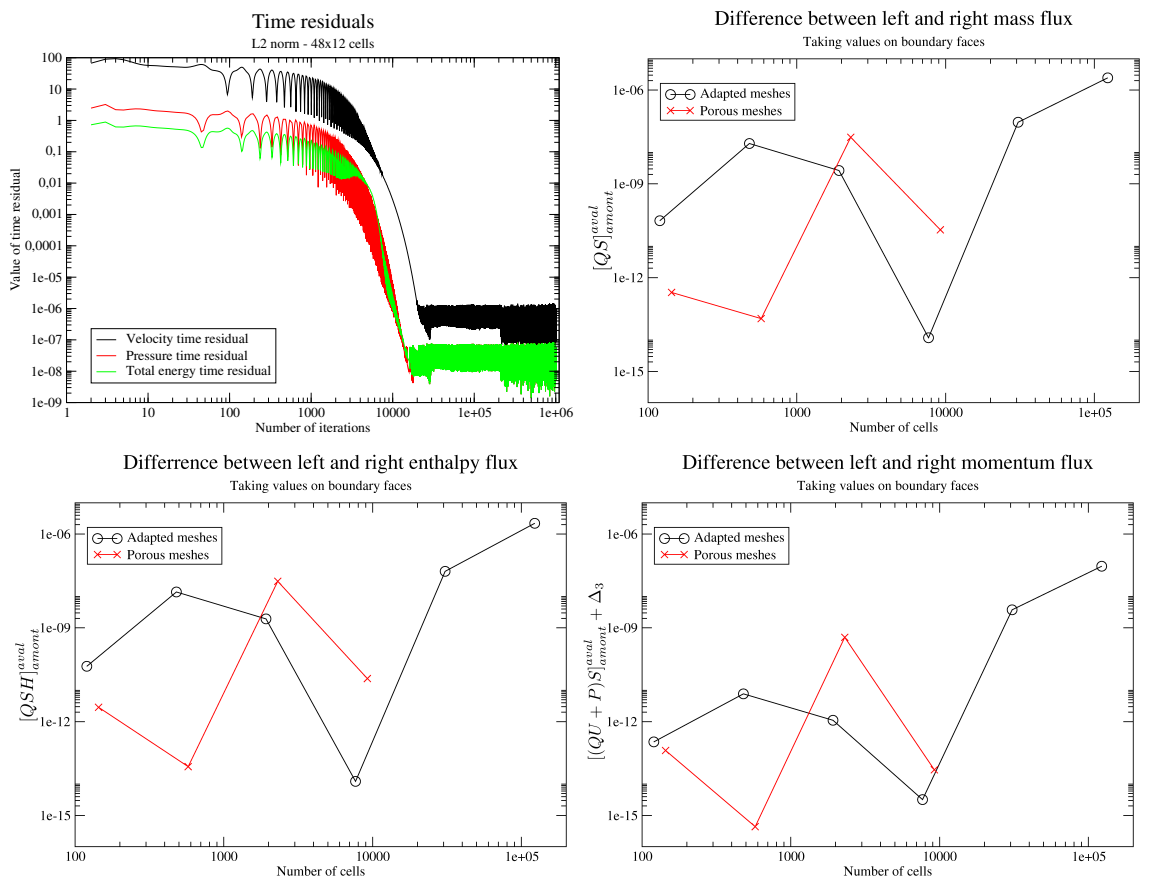

Fig. 3 Time residuals and value of $e(\boldsymbol{\varphi})$ for the adapted and porous meshes. The adapted meshes correspond to the black plots and the porous meshes to the red plots.

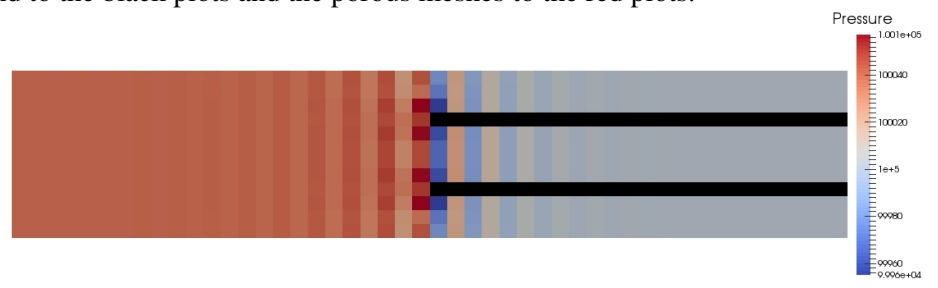

Fig. 4 Pressure contours $(\mathrm{Pa})$ for $48 \times 12$ porous mesh (black cells are solid).

between two simulations of different $M_{k}$ and $M_{l}$ configurations ( $k, l=1,2$ or 3$)$ on all $N_{\text {cells }}^{\phi}$ fluid cells for each discrete variable $\varphi_{i}=\left(\rho_{i}, P_{i}\right), i \in\left\{1, \cdots, N_{\text {cells }}^{\phi}\right\}$ (see Table 1,2 and 3) is defined as follows:

$$
E^{M_{k} M_{l}}=\max _{i \in\left\{1, \cdots, N_{\text {cells }}^{\phi}\right\}}\left|\varphi_{i}^{M_{k}}-\varphi_{i}^{M_{l}}\right| /\left|\varphi_{i}^{M_{l}}\right| .
$$

Here the domain measures are: $L=5$ and $h=1$. The deviations are rather weak $\left(\leq 10^{-3}\right)$. The porous formulation is robust w.r.t. standard computations. We note that the gaps are concentrated in the same area, near the upstream faces $(x=2.5)$. They are higher between $M_{3}$ and the other configurations. Current work aims at extending the integral formulation to incompressible viscous fluid flows governed 
by the Navier-Stokes equations. Viscous effects are taken into account thanks to a wall function which vanishes when the mesh is refined [2].

Table 1 Comparison of $M_{1}$ and $M_{2}$ configurations, $x$ and $y$ are the cell center coordinates.

\begin{tabular}{llll}
\hline Variables & $x$ & $y$ & $E^{M_{1} M_{2}}$ \\
\hline Density & 4.6875 & 0.1 & $2.3842 \times 10^{-6}$ \\
Pressure & 2.60417 & 0.1 & $1.6911 \times 10^{-8}$ \\
\hline
\end{tabular}

Table 2 Comparison of $M_{1}$ and $M_{3}$ configurations, $x$ and $y$ are the cell center coordinates.

\begin{tabular}{llll}
\hline Variables & $x$ & $y$ & $E^{M_{1} M_{3}}$ \\
\hline Density & 2.8125 & 0.1 & $1.5046 \times 10^{-3}$ \\
Pressure & 2.8125 & 0.1 & $2.205 \times 10^{-3}$
\end{tabular}

Table 3 Comparison of $M_{2}$ and $M_{3}$ configurations, $x$ and $y$ are the cell center coordinates.

\begin{tabular}{llll}
\hline Variables & $x$ & $y$ & $E^{M_{2} M_{3}}$ \\
\hline Density & 2.8125 & 0.1 & $1.5046 \times 10^{-3}$ \\
Pressure & 2.8125 & 0.1 & $2.2099 \times 10^{-3}$ \\
\hline
\end{tabular}

Acknowledgments The first author receives a financial support by ANRT through an EDF-CIFRE contract 2016/0728.

\section{References}

1. Archambeau, F., Hérard, J.M., Laviéville, J.: Comparative study of pressure-correction and godunov-type schemes on unsteady compressible cases. Computers \& Fluids 38, 1495-1509 (2009)

2. Colas, C., Ferrand, M., Hérard, J.M., Le Coupanec, E.: Approche intégrale pour la modélisation des écoulements en milieux encombrés - prise en compte des effets visqueux. Note interne 6125-3013-2016-17220-FR, EDF R\&D (2016)

3. Dubois, F.: Boundary conditions and the Osher scheme for the Euler equations of gas dynamics. Internal Report CMAP 170, Ecole Polytechnique, Palaiseau, France (1987)

4. EDF R\&D, http://code-saturne.org/cms/sites/default/files/docs/4.2/theory.pdf: Code_Saturne 4.2.0 Theory Guide (2015)

5. Ferrand, M., Hérard, J.M., Le Coupanec, E., Martin, X.: Schémas implicites dans une formulation intégrale pour la prise en compte d'obstacles immergés dans un fluide compressible. Note interne H-I83-2015-05276-FR, EDF R\&D (2015)

6. Hérard, J.M., Martin, X.: An integral approach to compute compressible fluid flows in domains containing obstacles. International Journal on Finite Volumes 12(1), 1-39, https://hal.archivesouvertes.fr/hal-01166,478/ (2015)

7. Le Coq, G., Aubry, S., Cahouet, J., Lequesne, P., Nicolas, G., Pastorini, S.: The THYC computer code. A finite volume approach for 3 dimensional two-phase flows in tube bundles. Bulletin de la Direction des études et recherches - Électricité de France. 61-76 (1989)

8. Martin, X.: Modélisation d'écoulements fluides en milieu encombré d'obstacles. Ph.D. thesis, Aix-Marseille Université, https://tel.archives-ouvertes.fr/tel-01235089/ (2015)

9. Toumi, I., Bergeron, A., Gallo, D., Royer, E., Caruge, D.: FLICA-4: A three-dimensional twophase flow computer code with advanced numerical methods for nuclear applications. Nuclear Engineering and Design 200, 139-155 (2000) 